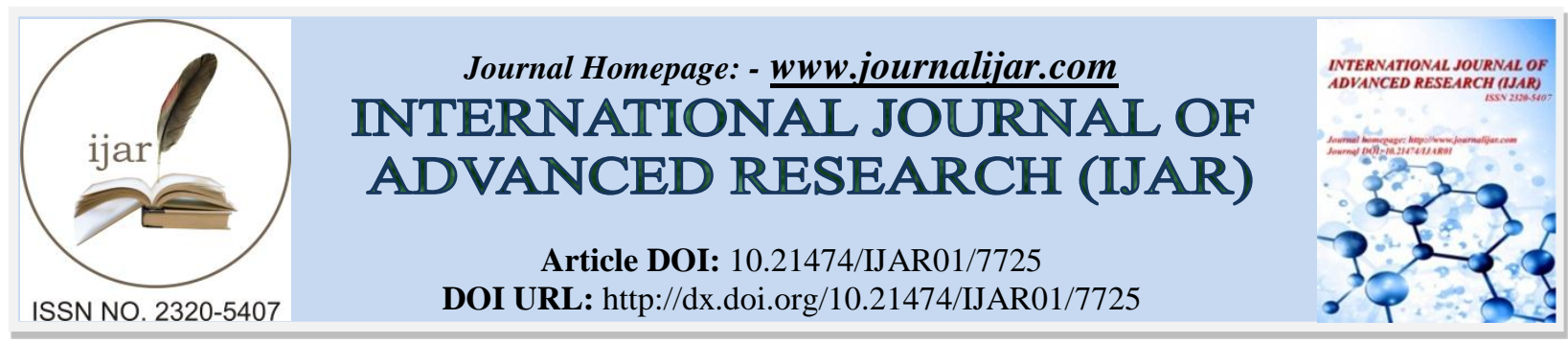

RESEARCH ARTICLE

\title{
POPLITEAL ARTERY INJURY CASE SERIES - AN INSTITUTIONAL EXPERIENCE.
}

\section{Prof. Dr. M. Bakthavatchalam, MS, Mch ${ }^{1}$, Dr. Devarajan. I MS ,Mch². Dr.K.Raja Dip.N.B (3)}

1. The Head of the Department, Department of vascular surgery, Tamil nadu Government Multisuper speciality Hospital Omandurar Estate, Chennai.

2. Assistant professor Institute of vascular surgery MMC, Chennai.

3. Senior resident Madras Medical College, Chennai.

\section{Manuscript Info}

Manuscript History

Received: 21 July 2018

Final Accepted: 27 August 2018

Published: September 2018

\section{Abstract}

Copy Right, IJAR, 2018,. All rights reserved.

\section{Introduction:-}

Arterial injuries of the lower limbs are common following RTA, resulting in amputation, lifestyle limitation, and mortality if not intervened appropriately (1)(2). In simple arterial injuries following revascularisation procedure, the results are generally good ranging more than 95\% limb salvage rates (3)(4),but in case of complex arterial injuries like popliteal artery injuries and multilevel vascular injuries, results in poor outcomes in terms of limb salvage which ranges from $60 \%$ to $70 \%$.The rate of amputation is very high in popliteal artery injuries because of limited collaterals in the below knee level (5).

Limb salvage in patients with popliteal artery injury eventually depend on the duration of ischemia, mode of injury, concomitant injuries and delayed or no revascularisation, or failed revascularisation. Timely intervention of these injuries is of great importance for better limb salvage (6). we did this retrospective study on 130 cases of popliteal artery injury patients who presented to our hospital , RGGGH , Institute of vascular injury between August 2010 and July 2015. All these patients were taken up for revascularisation procedure after clinical examination and doppler assessment. Amongst these 129 cases, 92 limbs were salvaged, 30 patients ended up in major amputation and 7 patients did not continued treatment following revascularisation procedure .

\section{Aim:-}

To present the incidence and various clinical presentations of popliteal artery injuries and limb salvage following revascularisation procedure at our hospital, RGGGH, Institute of vascular surgery between August 2010 and July 2015.

\section{Material and Methods:-}

Type of study

Retrospective analysis of prospective data

\section{Study Duration}

August 2010 to July 2015 
Sample size: 129

Inclusion criteria:

All the patients who underwent repair for popliteal artery injury at the Institute of Vascular Surgery both in the elective and emergency setting from August 2010 to July 2015 were included in the study

These patients were explained about the risk and complications of surgery including risk of limb loss, reperfusion injury and mortality. Patients who were willing for surgery, a high risk informed consent was taken and they were subjected to the planned procedure.

\section{Exclusion criteria:}

Patients with popliteal artery injury died before any intervention and those who did not consent to undergo revascularisation procedure were excluded from the study.

\section{Methodology:-}

Collection of data from case records - Patient details, History, clinical examination findings including Pulse status, ABPI, Co existing trauma details Assessment of time since injury till presentation at hospital The type of repair done, conduit used, patency, wound healing assessed Influence of factors in limb salvage assessed

Thorough physical examination of patients admitted with lower limb injuries by careful pulse examination and ankle brachial pressure measurement allows rapid assessment of the limb's vascular status for any vascular injuries(9). Diagnosis was made mostly by physical examination alone and by Doppler Ultrasonography or angiography in suspicious cases with "soft signs" such as palpable but diminished distal arterial pulses. (4)(12)

\begin{tabular}{|l|l|}
\hline Signs of ischemia Hard signs & Soft signs \\
\hline Absent distal pulses & Diminished distal pulse \\
\hline Palpable thrill or audible bruit & History of severe haemorrhage \\
\hline Actively expanding hematoma & Neurological deficit \\
\hline Active pulsatile bleeding & Proximity of wound to named vessel \\
\hline
\end{tabular}


In this retrospective study, we reviewed our 5-year experience with a subset of patients who had popliteal arterial injuries, being a high volume tertiary care centre, we serve as a referral unit 24 × 7 × 365 for all over Tamil Nadu and we also get cases from Andhra Pradesh and Karnataka.

Popliteal artery Injury confers high morbidity and mortality in our institution among the entire peripheral vascular trauma.

Time delay and severity of injury has been very crucial and also decisive in these cases where collaterals get occluded very easily.

\section{Treatment Principles}

Nonoperative Management

1. The presence of arterial injury in a traumatic extremity is itself not an indication for repair.

2. Stain et al reported on 45 extremity arterial injuries noted on routinely performed post-trauma angiography and treated initially without surgical exploration. Injuries included small, non-flow-limiting intimal defects and flaps, small pseudo aneurysms, and arteriovenous fistulae.

3. None of the injuries produced distal ischemia, nor was there free extravasation. Of these 45 injuries, 31 (69\%) were proximal to the knee or elbow. None of the 45 progressed to require operative intervention after a mean of more than 5 weeks of follow-up.

4. But a high index of suspicion for the possible development of a pseudo aneurysm or ischemic complication is necessary during a period of close clinical follow-up. Injuries identified on imaging that may be considered for observation include those that produce no active haemorrhage or distal ischemia, such as

5. Small intimal tears or flaps

6. Pseudo aneurysms

7. Arteriovenous fistulae

8. In these patients serial surveillance with duplex or CTA may be mandatory for the follow up of such patients. (4) 

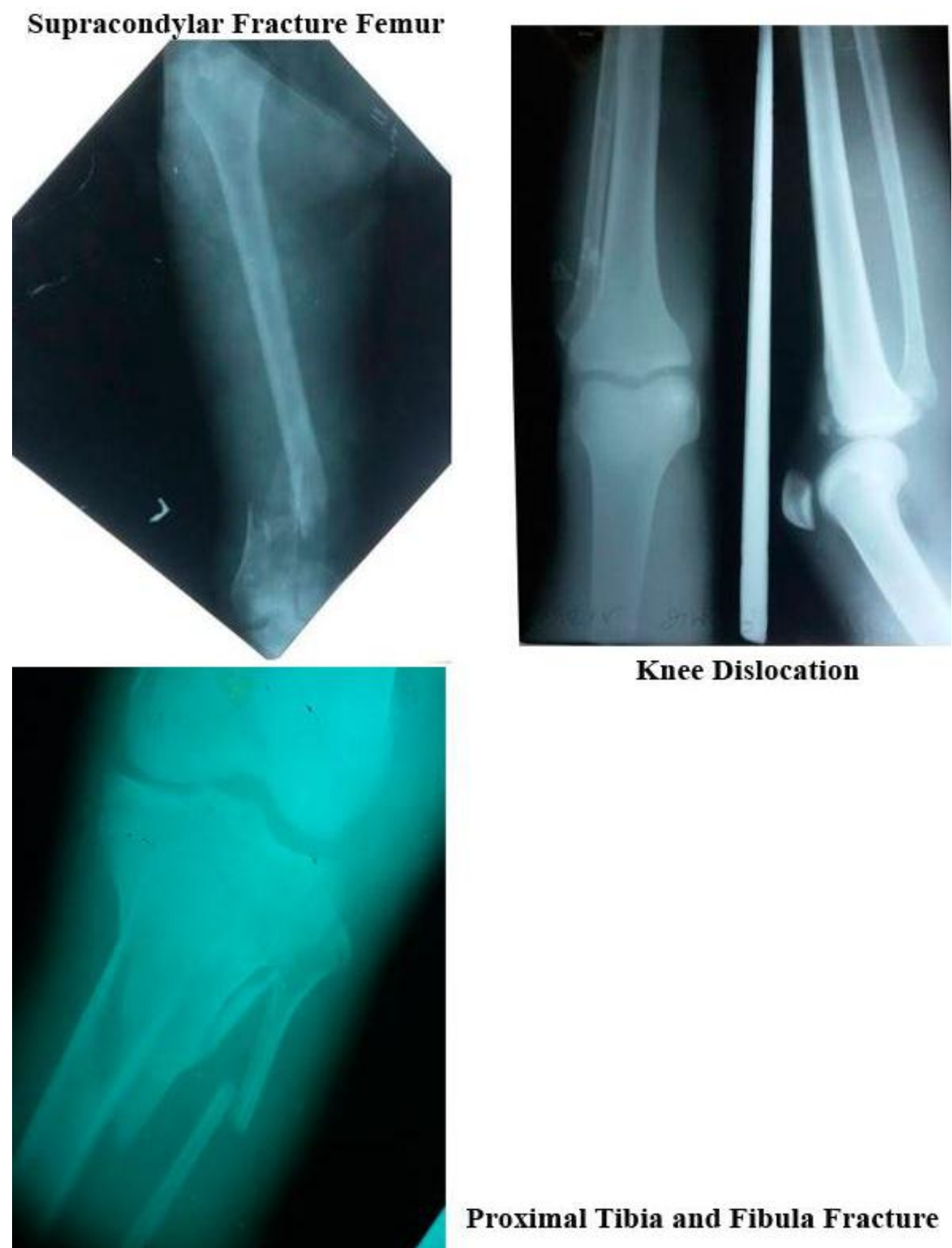

Knee Dislocation

\section{Proximal Tibia and Fibula Fracture}

Fig 1:-shows fracture femur, dislocated knee and both bone fracture leg. 


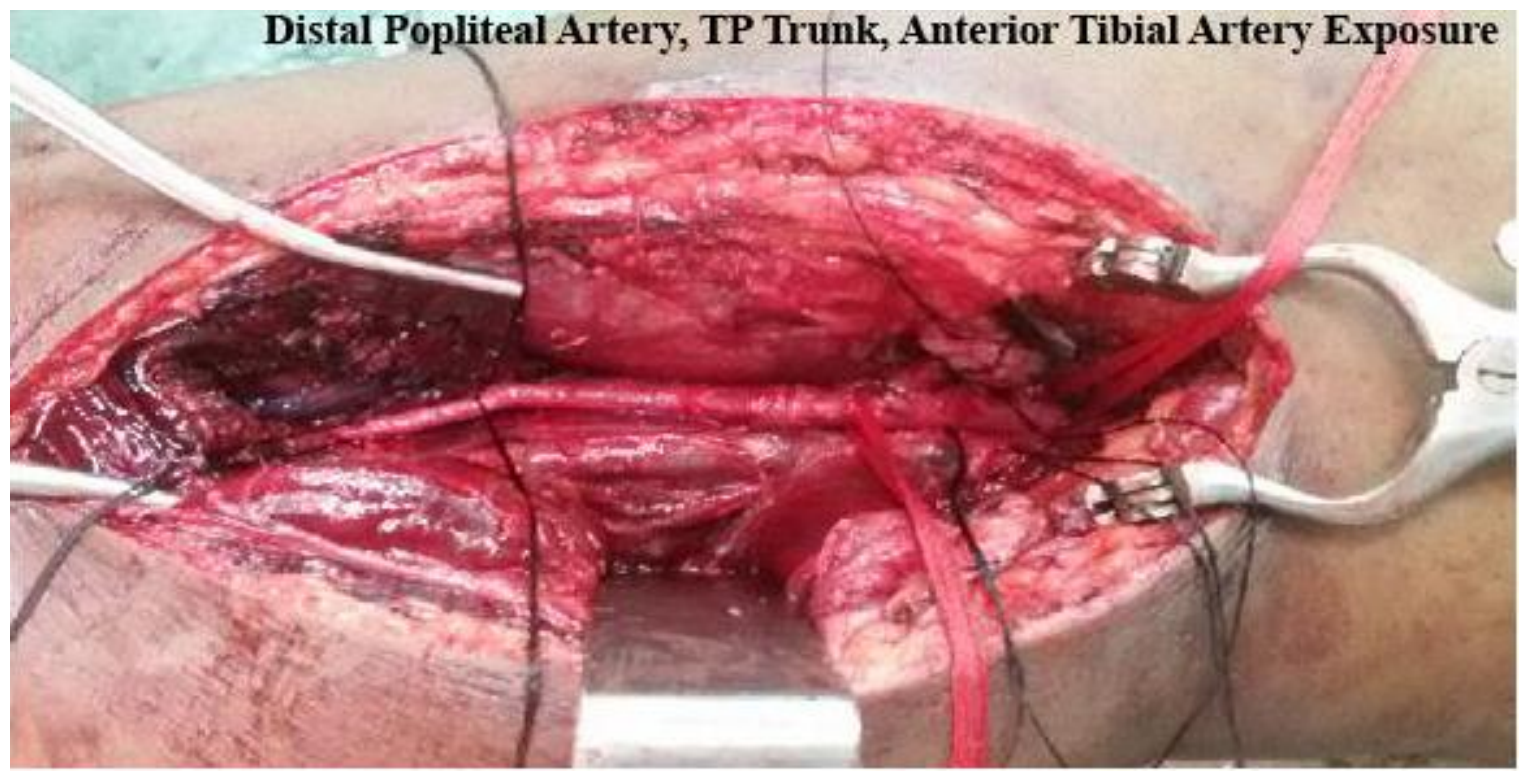

Fig 2:-Exposure of below knee arteries

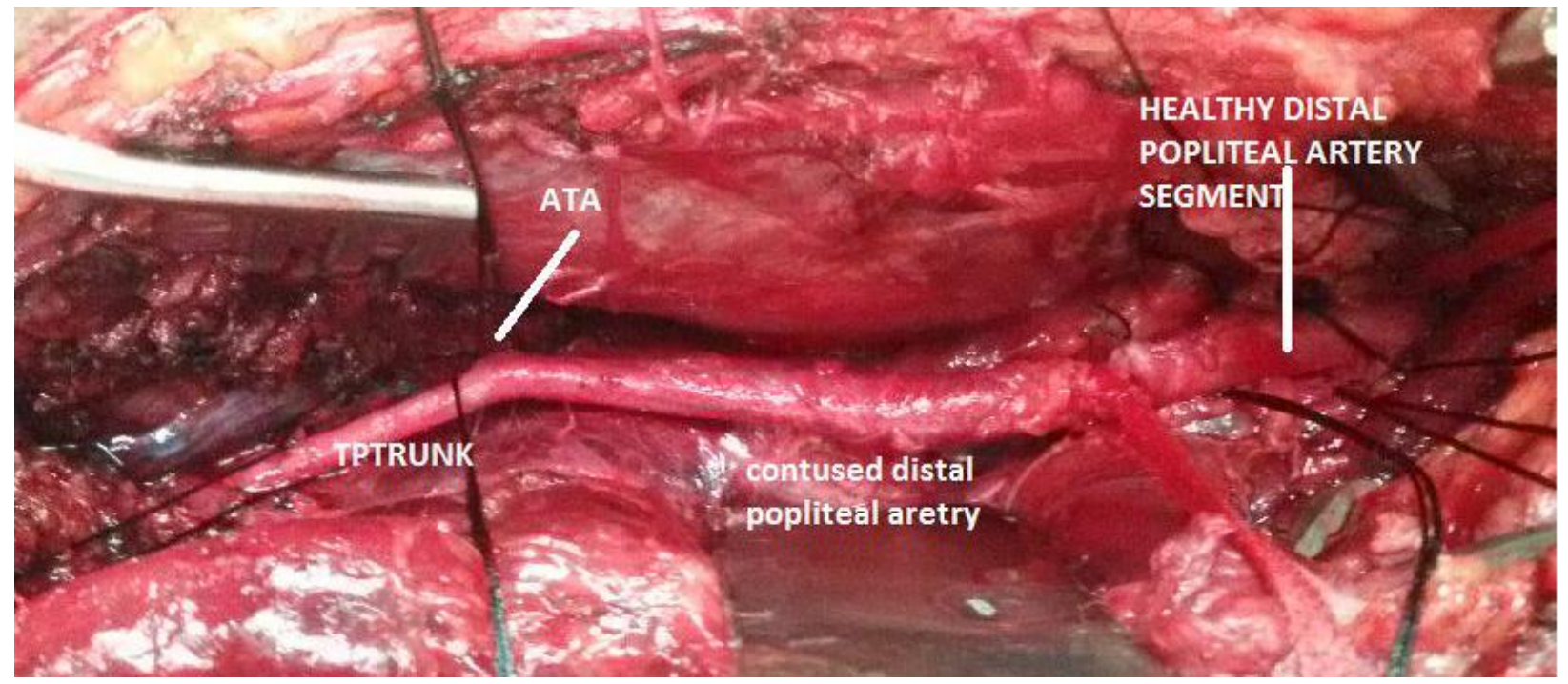

Fig 3:-Exposure of injured distal popliteal artery 


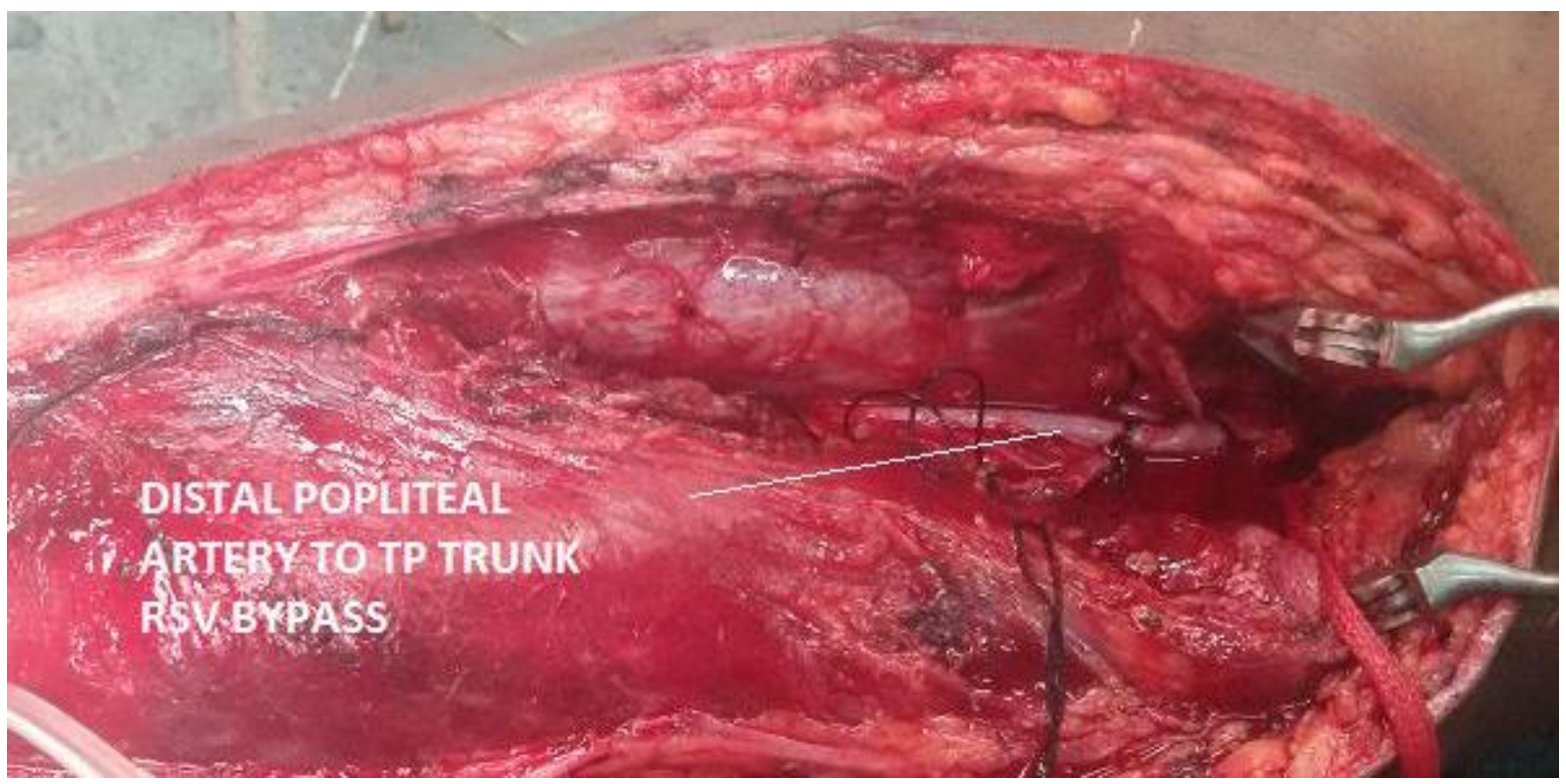

Fig 4:-Exposure of interposition graft repair in popliteal artery injury.

\section{Open Surgical Management}

Open surgical control and repair remain the mainstays of the management of most extremity vascular injuries

\section{Surgical Anatomy}

1. The popliteal artery originates at the adductor hiatus as the continuation of superficial femoral artery, throughout its course; the popliteal artery is located deep in the popliteal fossa along the posterior aspect of the femur, in proximity to the joint line, and the tibial plateau.

2. The artery is covered proximally by the semimembranosus muscle and in its mid portion by subcutaneous tissue. The artery continues distally to the upper calf where it terminates at the origin of anterior tibial artery at the triceps surae formed by the two heads of gastrocnemius muscle and the soleus muscle. Along its course the popliteal artery has 6 to 8 small geniculate branches, which are usually paired. These form a collateral network around the knee (4) .but during injury and acute occlusion these collaterals will not be sufficient to provide adequate distal flow.

3. The relationship of the popliteal artery to the muscles of the thigh and calf places it at risk for severe injury in the dislocation of the knee. During full extension, the popliteal artery is on tension across the back of the knee joint.

4. Knee dislocation stretches the popliteal artery over the posterior edge of the tibial plateau resulting in severe intimal injury or transaction.

5. The three tibial vessels have a variable origin. In $85 \%$ to $90 \%$ of individuals the popliteal artery bifurcates into anterior tibial and tibio-peroneal trunk. The posterior tibial and the peroneal artery arise 3 to $6 \mathrm{~cm}$ distally. In 10 $\%$ to $15 \%$ of the individuals the anterior and posterior tibial arteries arise at or above the knee joint line. (4) The popliteal and the tibial arteries are usually accompanied by a pair of veins.

\section{Clinical Feature $\mathbf{S}$ And Diagnosis}

1. Occlusion with thrombus and distal limb ischemia is the commonest presentation of patients with popliteal artery injury. Associated vein and nerve injuries results in poor functional outcome. Thrombosis of the popliteal artery with tibial vessels results in ischemia in the foot and leg. Dislocation of the knee joint produces a injury to the intima and delayed thrombosis.

2. Clinical examination is very crucial to identify popliteal or tibial vessel injuries. Any delay will result in limb or even life loss. When the distal pulses are absent, ABI measurement identifies distal perfusion, any index drop below 0.8 or a $20 \mathrm{~mm} \mathrm{Hg}$ drop in pressure compared to the normal limb indicate the need for further evaluation. 
3. Duplex has a limited role in acute arterial trauma, even when used by experienced persons it will not obviate the need of arteriography, arteriography is accurate in predicting the arterial injury but time consuming. Intraoperative angiogram is also accurate and is very useful.

4. Open surgical procedures should be performed preferably in OT table with arteriography facilities. I t solves two issues

\section{On table angiography can be performed}

Associated bony injury can be managed concomitantly.

1. The patient should be prepared and draped widely to facilitate exposure, typically circumferentially in the case of extremity injuries.

2. If the need for a vein graft is anticipated, the proposed harvest site should be included in the prepared operative field. Conventional practice is to obtain saphenous vein grafts from uninjured extremities to preserve collateral venous drainage.

3. This dogma is not supported by good-quality data, however, and it may be appropriate to harvest conduit from an injured extremity if there is no venous injury

\section{Steps In Repair And Reconstruction}

The general principles are

1. Establishing proximal and distal vascular control

2. Restoring in-line flow to a suitable outflow bed are the same for the treatment of traumatic lesions as for any other vascular defect.

3. Because traumatic vascular injuries frequently present with significant hemorrhage, the ability to achieve vascular control expeditiously is paramount

4. Pneumatic operative tourniquets, placed in a sterile or unsterile fashion, can be used to provide rapid control of inflow until formal arterial control proximal to a bleeding injury can be obtained.

5. To ensure adequate exposure for control and repair, incisions are typically made longitudinally

6. Directly over the target vessel proximal and distal to the injury.

7. Once vascular control is achieved, the incisions can be extended as needed to expose the zone of vascular injury.

8. In some cases of extremity injury, control may need to be obtained proximal to the extremity itself

9. Local anticoagulation with heparinised saline injection directly into the injured vessel proximal and distal to the injury may be used to prevent local thrombosis.

10. Wide debridement of contaminated and nonviable tissue within the zone of vascular injury should be performed.

11. During debridement, an assessment of the availability of healthy tissue to cover the vascular repair should be made, as graft or repair coverage is essential to prevent desiccation and possible anastomotic breakdown and subsequent hemorrhage

12. The coordination with other departments is necessary for proper tissue coverage.

13. Intimal injury can extend beyond what is appreciated by an injured vessel's outward appearance. Before heparinisation and repair, the inflow and outflow vessels should undergo gentle balloon catheter thrombectomy to confirm patency

14. A $\mathrm{n}$ assessment of forward and back bleed should be assessed subjectively

If the patient's physiological status permits procedures to establish distal flow should be performed in all cases. Acute ligation of an injured extremity artery may be the most feasible acute option in some cases. Sometimes this may be done with a plan for formal revascularization when the patient's overall condition stabilizes. [43]

Three options exist for repair.

1. A short injured segment can be resected circumferentially and enough of the vessel freed proximally and distally to perform a spatulated end-to-end anastomosis of the injured vessel.

2. A single arteriotomy can be débrided and a vein patch angioplasty performed.

3. In many cases, however, neither of these "simple" options is feasible, and a vein interposition graft of appropriate length must be performed. 
All open traumatic wounds are considered contaminated, and the preferred conduit for repair of vascular injuries is autogenously saphenous vein harvested from an uninjured extremity.

Infections involving prosthetic grafts are notoriously difficult to manage, resulting in high rates of graft explantation and amputation.

These issues are exacerbated when there is inadequate soft tissue coverage of the graft.

The prosthetic arterial grafts most at risk for thrombosis were those placed in smaller vessels. A short-segment prosthetic graft may be considered to repair uncontaminated (typically blunt) arterial injuries to large vessels, such as the axillary and common femoral arteries, where size match with the saphenous vein may be a problem.

\section{Temporary Shunting}

It is a temporizing measure allowing distal perfusion during fracture stabilization before formal vascular repair or as an adjunct to damage control management of a patient unable to undergo definitive repair

Arterial shunt dwell times have been in the range of 1 to 3 hours, with patency rates approaching $100 \%$ in the absence of the use of systemic anticoagulation

Arterial shunts placed in the forearm and tibial vessels have poor patency (12\% vs. $86 \%$ for proximal shunts) as presented in a recent military report (4)

\section{Venous Repair}

\section{It is a controversial subject.}

There has been no consistent association between major venous ligation and eventual amputation. The high incidence of acute thrombosis of venous repairs is supported by data from Smith et al, who reported $45 \%$ occlusion rate at 72 hours.

The development of limb oedema is seen as the primary potential adverse outcome when a major extremity venous injury is ligated. The development of edema is more common when ligation is performed at the popliteal than at the femoral level, with transient swelling seen in up to

[45]

$90 \%$ of ligated popliteal and $50 \%$ of ligated femoral injuries. Repair reduces the transient edema by about half, to $50 \%$ and $29 \%$, respectively.

But most of these cases resolve spontaneously.

Venous thromboembolism is one more important complication of ligated veins.

There is no clear indication of whether the deep venous thromboses seen in this population are the result of the venous ligation, however, and the rates of pulmonary embolism are similar between patients undergoing ligation and repair.(13)

The decision to ligate or repair depends on

\section{General condition}

Time required for repair

End-to-end anastomosis, Lateral suture venorrhaphy, Patch venoplasty, and Interposition grafting are all viable options for repair, depending on the anatomy of the injury.

Regardless of whether ligation or repair is performed, a period of leg elevation and gentle compression can help reduce the incidence of postoperative edema and possibly eventual venous insufficiency 
Surgical Considerations The most appropriate exposure of the injured popliteal artery is through a separate medial above-knee and below-knee incisions. Although they do not allow direct access to the retrogeniculate popliteal artery, These incisions allow proximal and distal vascular control and extension to the SFA and crural arteries, and they permit a number of options for arterial repair. If the injury is not directly repaired (i.e., retrogeniculate injury), there is a theoretical risk of late pseudo aneurysm formation if the injured but excluded segment does not thrombosehe medial exposure of the popliteal artery directly behind the knee should be avoided because the necessary division of the semimembranosus and semitendinosus tendons invites considerable morbidity during rehabilitation.

A posterior approach to the popliteal may be used to treat an arterial injury that is known to be focal and behind the knee, but most injured patients cannot be safely placed in the required prone position and it is frequently difficult to know the true extent of arterial injury preoperatively.

Because of the relatively fixed location and nature of the exposure of the popliteal artery, primary anastomosis is not typically possible, and the majority of injuries are repaired with an interposition graft.(15)

Almost one third of the patients with popliteal artery injury undergo amputation. Several attempts of revascularisation had been reported eventually resulting in amputation due to poor wound healing. Much care should be taken in selecting the patients for revascularisation.

Vascular injuries in penetrating wounds are usually easy to diagnose as there will be absent pulses and with clear signs of limb ischemia. It is often difficult to diagnose a popliteal artery injury in blunt trauma patients. Posterior dislocations of the knee, is very likely to injure the popliteal artery. (6)

The time of preoperative evaluation should be as short as possible to minimize ischemia time and thus prevent potential necrotic changes. According to a recent study, in the management of extremities with soft signs of vascular injury, ankle brachial pressure index measurement should be the first-line diagnostic choice to exclude arterial injury in $99.5 \%$ of patients (7) and to avoid unnecessary examination.

Baseline characteristics:

\begin{tabular}{|l|l|l|}
\hline Parameters & Number of cases & Cases in percentage \\
\hline Upper limb arterial trauma & 138 & $40 \%$ \\
\hline Lower limb arterial trauma & 185 & $54 \%$ \\
\hline Abdominal arterial trauma & 22 & $6 \%$ \\
\hline Femoral artery injury & 26 & $14 \%$ \\
\hline Popliteal artery injury & 129 & $69.7 \%$ \\
\hline Tibial artery injury & 30 & $16.2 \%$ \\
\hline Male & 114 & $88.4 \%$ \\
\hline Female & 15 & $11.6 \%$ \\
\hline Proximal popliteal injury & 25 & $19.4 \%$ \\
\hline Mid popliteal injury & 40 & $31 \%$ \\
\hline Distal popliteal injury & 64 & $49.6 \%$ \\
\hline Presentation before 24hrs of trauma & 104 & $80.6 \%$ \\
\hline Presentation after 24hrs of trauma & 25 & $19.4 \%$ \\
\hline Limb salvaged & 92 & $71.3 \%$ \\
\hline Major amputation & 30 & $23.25 \%$ \\
\hline $\begin{array}{l}\text { Discontinued treament after } \\
\text { procedure }\end{array}$ & 7 & $5.42 \%$ \\
\hline
\end{tabular}

\section{Results:-}

In this study, we had 138 cases of upper limb arterial trauma which constituted $40 \%$ of the trauma at our institution. 185 cases $(54 \%)$ comprising of lower limb arterial trauma and $22(6 \%)$ accounting for iliac and major abdominal arterial injuries. Popliteal artery injury was the most common arterial injury which constituted almost $70 \%$ of cases. Popliteal artery injury accounted for $37 \%$ of total arterial trauma in the institution. This is much more than what has been described in literature. In our experience, isolated tibial artery injury outnumbered femoral artery injuries (30 Vs 26 cases). 
Over the years in our institution, we have seen an almost equal distribution of popliteal artery injury based on segments. The distal popliteal segment is more frequently injured compared to other segments. The 5 year statistics reveals that patients more commonly presented less than $24 \mathrm{hrs}$ hours after injury. The majority of delayed presentation had been referred from neighboring towns sometimes even from towns of neighboring states.

In this study, amongst the 129 cases of popliteal artery injury patients who undergone revascularisation , 92 patients limbs were salvaged after successful revascularisation by interposition graft bypass, in the later group 84 patients arrived within $24 \mathrm{hrs}$ of arterial injury. 25 patients presented after $24 \mathrm{hrs}$ of arterial injury, amongst these 17 ended up in major amputation after revascularisation procedure. 7 patients got discharged at request, discontinued treatment and follow up after surgical revascularisation procedure.

Amongst the patients (30) who ended up in major amputation after revascularisation procedure, indication for amputations were graft thrombosis in 7 cases(23.33\%), wound infection and septicemia in 13 cases(43.33\%) and pseudoaneurysm formation with blow out in 5 cases(16.67\%).

\section{Discussion:-}

In our institution, popliteal artery injuries are the commonest lower limb arterial injuries which accounts for about $70 \%$ of cases, unlike the experience of singh kohli study in jammu(1)(10)(14). Popliteal artery injury accounted for $37 \%$ of total arterial trauma in our institution. This is much more than what has been described in literature. In our experience, isolated tibial artery injury outnumbered femoral artery injuries (30 Vs 26), this is again contraversial with respect to singh kohli study.

Extremity vascular injury occurs in younger age group and male sex preponderance noted . we have seen an almost equal distribution of popliteal artery injury based on segments. The distal popliteal segment is more frequently injured compared to other segments, which is not specified in any of the current articles.

The 5 year statistics reveals the patient more commonly presented within 24 hours after injury(3).

Most of our patients usually come with poly-trauma and are due to high velocity accidents. The orthopaedic injury is very commonly associated with our patients in the study accounting more than $90 \%$. Patients who reported within $24 \mathrm{hrs}$ after the injury, had better limb salvage rate (87.5\%) when compared to patients who reported after $24 \mathrm{hrs}$ of injury $(32 \%)$. This is in view of advanced ischemia and polytrauma . 7 patients who undergone procedure with interposition RSV graft, discontinued treatment and did not come for follow up(5.38\%). Remaining patients were managed with interposition graft repair using great saphenous vein from the opposite leg, in selected cases from the same leg in view of both leg injuries. Amongst the patients who ended up in major amputation, the causative factors were graft thrombosis, wound infection, septicemia and pseudoaneurysm formation.

\section{Conclusion:-}

From our institutional experience between August 2010 and July 2015, on 129 trauma victims who sustained popliteal artery injuries, the predictive factors of limb salvage are time of presentation following injury, severity of ischemia, associated soft tissue and bony injuries(5), timely repair with vascular reconstruction(11). Delayed presentation and polytrauma are associated with worst prognosis.

\section{Bibliography:-}

1. Singh G Kohli A, Management of extremity vascular trauma: Jammu experience. Asian Cardiovasc Thorac Ann. 2008

2. Andersen CA Mullenix PS, Steele SR, , Starnes BW, Salim A, Martin MJ. Limb salvage and outcomes among patients with traumatic popliteal vascular injury: an analysis of the National Trauma Data Bank. J Vasc Surg. 2006

3. Ebner JA, Giovannetti R Zaraca F, Ponzoni A, Stringari C, Ebner H. Lower extremity traumatic vascular injury at a level II trauma center: an analysis of limb loss risk factors and outcomes. Minerva Chir. 2011

4. Rutherford's text book of Vascular Surgery 8th edition.

5. Antoniou I, Panoussis Andrikopoulos V, P. Arterial injuries associated with lower-extremity fractures. Cardiovasc Surg. 1995

6. ER Frykberg. Popliteal vascular injuries. Surg Clin North Am. 2002 
7. Yanar H, Kuzkaya M Kurtoğlu M, Dolay K, Karamustafaoğlu B,. The role of ankle brachial pressure index in the diagnosis of peripheral arterial injury. Ulus Travma Acil Cerrahi Derg. 2009 Norman and rich vascular trauma 2nd edition

9. Pipinos I, Agnostopolous P, Carter Y Guerrero A, Gibson K, Kralovich KA et al. Limb loss following lower extremity arterial trauma: what can be done proactively? Injury. 2002 10. Doley R, Venkatesan R Menakuru SR, Behera A, Jindal R, Kaman L,. Extremity vascular trauma in civilian population: a seven-year review from North India. Injury. 2005

10. Kostic DM, Dragas MV Davidovic LB, Cinara IS, Ille T, , Markovic DM. Civil and war peripheral arterial trauma: review of risk factors associated with limb loss. Vascular. 2005

11. Callcut RA, Acher CW, Hoch J, Tefera G, Turnipseed W, Mell MW. Impact of intraoperative arteriography on limb salvage for traumatic popliteal artery injury. J Trauma. 2009

12. Quan RW, et al: The effect of vein repair on the risk of venous throm- boembolic events: a review of more than 100 traumatic military venous injuries. J Vasc Surg 47:571-577, 2008.

13. Franz RW, et al: A 5-year review of management of lower extremity arterial injuries at an urban level I trauma center. J Vasc Surg 53:1604- 1610, 2011.

15. McDowell HA, Diethelm AG Reynolds RR,. The surgical treatment of arterial injuries in the civilian population. Ann Surg. 